\title{
Single molecule DNA analysis based on atomic-controllable nanopores in covalent organic frameworks
}

\author{
Xiao-Lei Xing ${ }^{\dagger, 1}$, Qiao-Bo Liao ${ }^{\dagger, 1}$, Saud Asif Ahmed ${ }^{1}$, Dong-Ni Wang ${ }^{1}$, Shi-Bin Ren ${ }^{3}$,

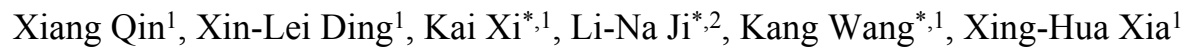

1. State Key Laboratory of Analytical Chemistry for Life Science, School of Chemistry and Chemical Engineering, Nanjing University, Nanjing 210023, China

2. State Key Laboratory of Pharmaceutical Biotechnology, School of Life Sciences, Nanjing University, Nanjing 210023, China

3. School of Pharmaceutical and Materials Engineering, Taizhou University, 317000, Taizhou, P.R. China

E-mail: xikai@nju.edu.cn (K. Xi); jilina@nju.edu.cn (L.N. Ji); wangkang@nju.edu.cn (K. Wang)

$\dagger$ These authors contributed equally.

\section{Table of Contents}

S1. Materials and Methods

S2. Supplementary Figures and Discussion

Figure $\mathrm{S}_{1}$ to Figure $\mathrm{S}_{4}$

S3. Supplementary Tables

Table $\mathbf{S} 1$ and Table S2

References 


\section{S1. Materials and Methods}

\section{Chemicals}

Reagents for COFs synthesis: 4',4"',4"'",4"'"'--(1,2-ethenediylidene) tetrakis[1,1'biphenyl]-4-carbox-aldehyde $\quad$ (ETBC， 97\%), 4,4',4",4"'-(ethene-1,1,2,2-tetrayl)-

tetraaniline (ETTA, 97\%) and 4,4',4",4'"-(ethene-1,1,2,2-tetrayl)-tetrabenzaldehyde (ETTB, 98\%) were received from Jilin Extension Technology Co., Ltd. O-dichlorobenzene (o-DCB), n-butanol, acetic acid, tetrahydrofuran (THF), 1,4-dioxane and mesitylene were all analytical grade. All chemicals were used without further purification.

Reagents for nanopore fabrication and DNA transport: Hexadecyl trimethyl ammonium bromide (CTAB, 99\%) was purchased from Aladdin. Rhodamine 6G (R6G, $99 \%$ ), tetramethylrhodamine-5 maleimide (TMRM, $\geq 85 \%$ ) and calf thymus DNA (ctDNA, MW: $\sim 15$ million, $\sim 50 \mathrm{k}$ bp) were purchased from Sigma-Aldrich. An 80 bp ssDNA (DNA-80) was synthesized by Shanghai Sangon (China) with HPLC purification (the sequence was list in Tab. S1). TE buffer (10 mM Tris-HCl, 1 mM EDTA, pH 8.0) was used for the translocation experiment. $\mathrm{LiCl}(\geq 97 \%)$ and other reagents were analytical grade. All aqueous solutions were prepared using water from a Millipore system $\left(18 \mathrm{M} \Omega \cdot \mathrm{cm}^{-1}\right)$.

\section{COFs synthesis and exfoliation}

Synthesis of COF-1.1 $16.7 \mathrm{mg}(0.06 \mathrm{mmol})$ of ETTC, $23.6 \mathrm{mg}(0.06 \mathrm{mmol})$ of ETTA, $0.4 \mathrm{~mL}$ of o-DCB/n-butanol $(7: 3, \mathrm{v} / \mathrm{v})$ and $0.05 \mathrm{~mL}$ of $12 \mathrm{M}$ aqueous acetic acid solution were charged into a $2 \mathrm{~mL}$ glass tube. The mixture was degassed by three freezepump-thaw cycles. The glass tube was flame-sealed under vacuum and then heated at $120{ }^{\circ} \mathrm{C}$ for 3 days. After cooling, the mixture was filtered through a $0.22 \mu \mathrm{m}$ PTFE membrane and washed with THF several times to remove unreacted monomers, the catalyst and solvent. The filter cake was further purified by Soxhlet extraction using THF for 24 hours, followed by drying under supercritical $\mathrm{CO}_{2}$ flow. 
Synthesis of COF-1.3 $44.9 \mathrm{mg}(0.06 \mathrm{mmol})$ of ETBC, $23.6 \mathrm{mg}(0.06 \mathrm{mmol})$ of ETTA, $0.4 \mathrm{~mL}$ of o-DCB/n-butanol (7:3) and $0.05 \mathrm{~mL}$ of $12 \mathrm{M}$ aqueous acetic acid solution were charged into a $2 \mathrm{~mL}$ glass tube. The following degassing, reacting and purifying steps were the same with that of COF-1.1.

The obtained powders were characterized by FTIR (Fourier transform infrared spectroscopy, Nicolet iS50). PXRD (Powder X-ray diffraction) patterns of the samples were recorded on a Bruker D8 Advance X-ray diffractometer with $\mathrm{Cu}-\mathrm{K} \alpha$ radiation $(\lambda=$ $0.15418 \mathrm{~nm})$ and a step size of 0.02 .

Exfoliation of COF nanosheets $0.5 \mathrm{mg}$ of the COF powder was dispersed in $5 \mathrm{~mL}$ $\mathrm{KCl}$ solution $(10 \mathrm{mM})$, then $100 \mu \mathrm{L} \mathrm{CTAB}(1 \mathrm{mM})$ was added into the mixture followed by ultrasonic exfoliation $(\mathrm{XO}-1000 \mathrm{D}, 1 \mathrm{~kW})$ for one hour ${ }^{[\mathrm{s} 1, \mathrm{~s} 2]}$. The exfoliated flakes were then purified twice by centrifugation at 1,000 rpm for $10 \mathrm{~min}$ and re-dispersed in $10 \mathrm{mM}$ $\mathrm{KCl}$ for later use. AFM (Atomic force microscopy, ICON, Bruker) and TEM (Transmission electron microscopy, JEM-2100, JEOL) were used to characterize the morphology of the obtained COF nanosheets.

\section{Fabrication of COF nanosheet-covered quartz nanopipettes}

Quartz capillaries with inner diameter of $0.7 \mathrm{~mm}$ and outer diameter of $1.0 \mathrm{~mm}$ (Q10070-10, Sutter Instrument) were pulled using a micropipette puller (P-2000, Sutter Instrument). The parameters used in the fabrication are shown below:

Quartz: HEAT=850, FIL=2, VEL=22, DEL=160, PUL=250.

The quartz nanopipette was backfilled with $10 \mathrm{mM} \mathrm{KCl}$ and dipped into the $\mathrm{COF}$ nanosheets dispersion solution containing $0.1 \mathrm{mg} / \mathrm{mL}$ COF nanosheets and $10 \mathrm{mM} \mathrm{KCl}$. Then, two $\mathrm{Ag} / \mathrm{AgCl}$ wire electrodes were placed on the inside and outside of the quartz nanopipette, and a bias voltage of $-1 \mathrm{~V}$ was applied across the quartz nanopipette. Because the CTAB modified COF nanosheets were positively charged, the electrophoretic force then push COF nanosheets moving toward the orifice of the nanopipette. The current-time 
(I-t) curve during the electrophoresis was recorded. Usually, an obvious current drop could be observed within $200 \mathrm{~s}$, indicating the covering of COF nanosheet on the tip of the nanopipette. Then, the obtained COF-covered quartz nanopipettes were stored in the air or

directly used for subsequent measurement. Some of the nanopipette were thoroughly dried and characterized by scanning electron microscopy (SEM, FE-SEM S-4800, Hitachi).

\section{Electrochemical Instruments}

An Axopatch 200B patch clamp amplifier (Molecular Devices, Inc.) is used to record the ionic current in the single cell configuration with a sampling rate of $500 \mathrm{kHz}$ and lowpass filter of $10 \mathrm{kHz}$. The DNA solution was put in the trans side (outside of the nanopipette) and the same buffer solution without DNA was filled in the cis side (inside of the nanopipette). $\mathrm{Ag} / \mathrm{AgCl}$ electrodes are inserted in both cis and trans sides, then connected to the Axopatch 200B with a digitizer (Digidata 1550B). All measurements were taken inside a dark Faraday cage. A positive potential was applied to the cis electrode inside the pipette in order to drive DNA passing through the COF nanopores by electrophoretic force. 


\section{S2. Supplementary Figures and Discussion}
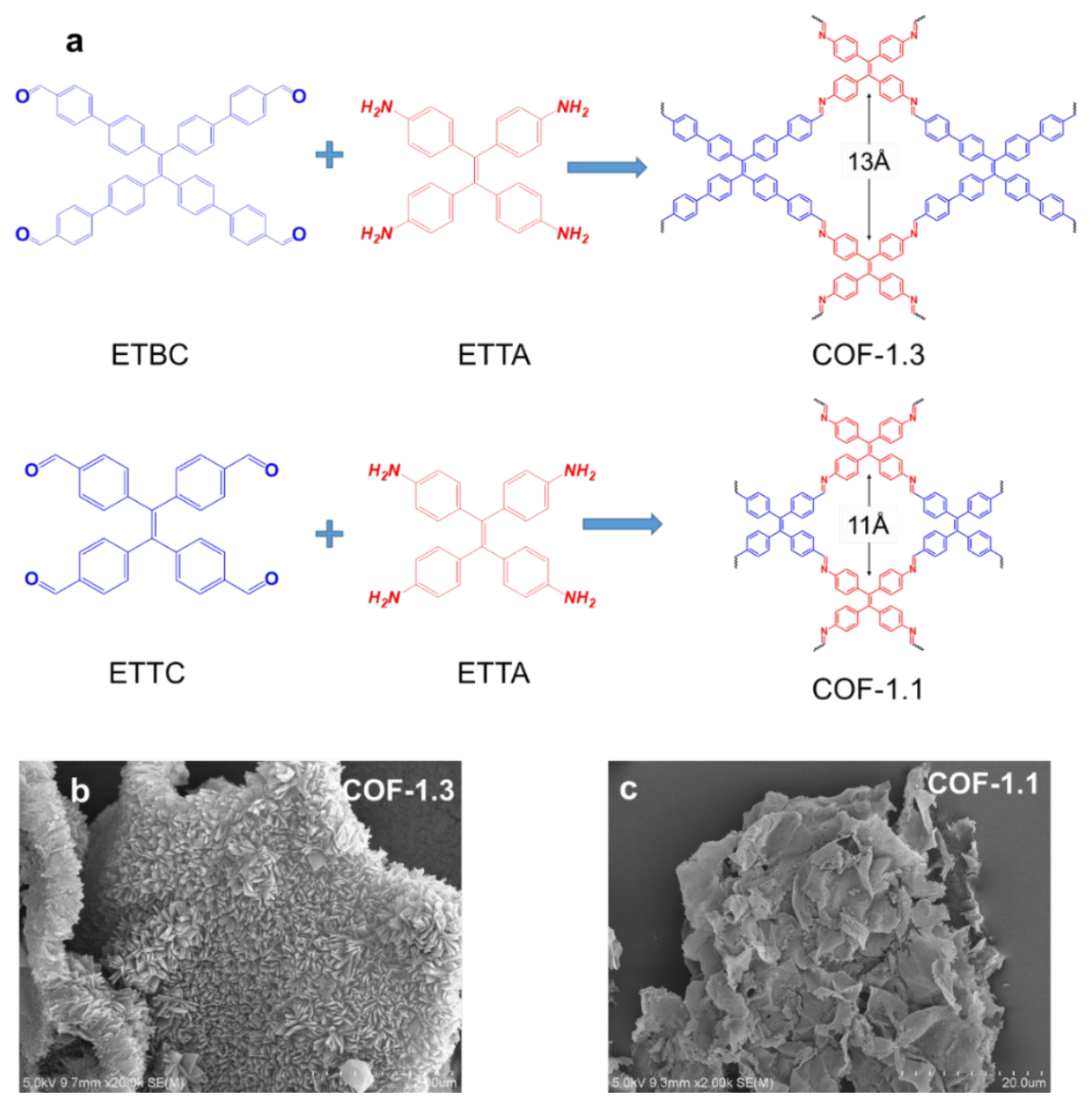

Figure S1. (a) Synthetic schemes of COF-1.3 and COF-1.1. The monomers were dissolved in $12 \mathrm{M}$ aqueous acetic acid solution, flame-sealed under vacuum, and then heated at $120^{\circ} \mathrm{C}$ for 3 days. (b) SEM image of COF-1.3, (c) SEM image of COF-1.1. 

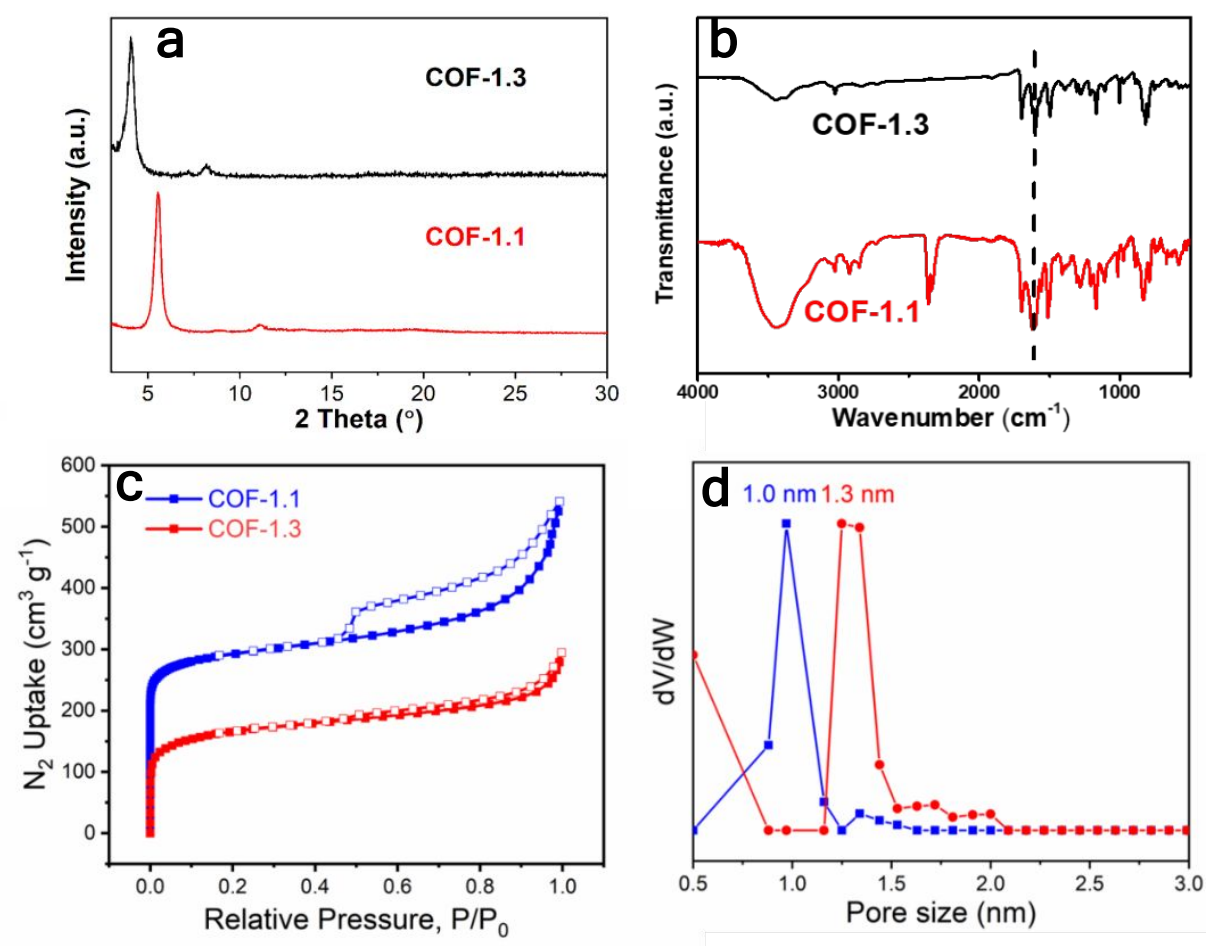

Figure S2. The XRD patterns (a) and FTIR spectra (b) of COF-1.1 and COF-1.3. The adsorption peak at $\sim 2400 \mathrm{~cm}^{-1}$ in the FTIR spectrum of COF-1.1 may correspond to the residual tetrahydrofuran (THF) from the preparation process [s3, s4]. (c) $\mathrm{N}_{2}$ sorption isotherms and (d) pore size distribution of COF-1.1 and COF-1.3.

The PXRD patterns for COF-1.1 and COF-1.3 show strong peaks at $2 \theta=5.6^{\circ}$ and $4.1^{\circ}$, respectively, indicating that both COFs have high crystallinity (Fig. S2a). The full width at half-maximum (FWHM) of the main reflection peaks of COF-1.1 $\left(0.442^{\circ}\right)$ resembles that of COF-1.3 $\left(0.474^{\circ}\right)$, suggesting that their crystallinities are similar. In the FTIR measurement, new peaks at $\sim 1600 \mathrm{~cm}^{-1}$ assigned to the stretching mode of imine bond show up for both COF-1.1 and COF-1.3 (Fig. S2b). By fitting the $\mathrm{N}_{2}$ isotherms of COFs with Brunauer-Emmett-Teller (BET) theory (Figure S2c), the specific surface areas of COF-1.1 and COF-1.3 are determined to be 1129 and $613 \mathrm{~m}^{2} / \mathrm{g}$, respectively. Calculated by the nonlocal density functional theory (NLDFT), the main pore sizes of COF-1.1 and COF-1.3 are 1.0 and $1.3 \mathrm{~nm}$ (Figure S2d), agreeing well with the predicted results. 

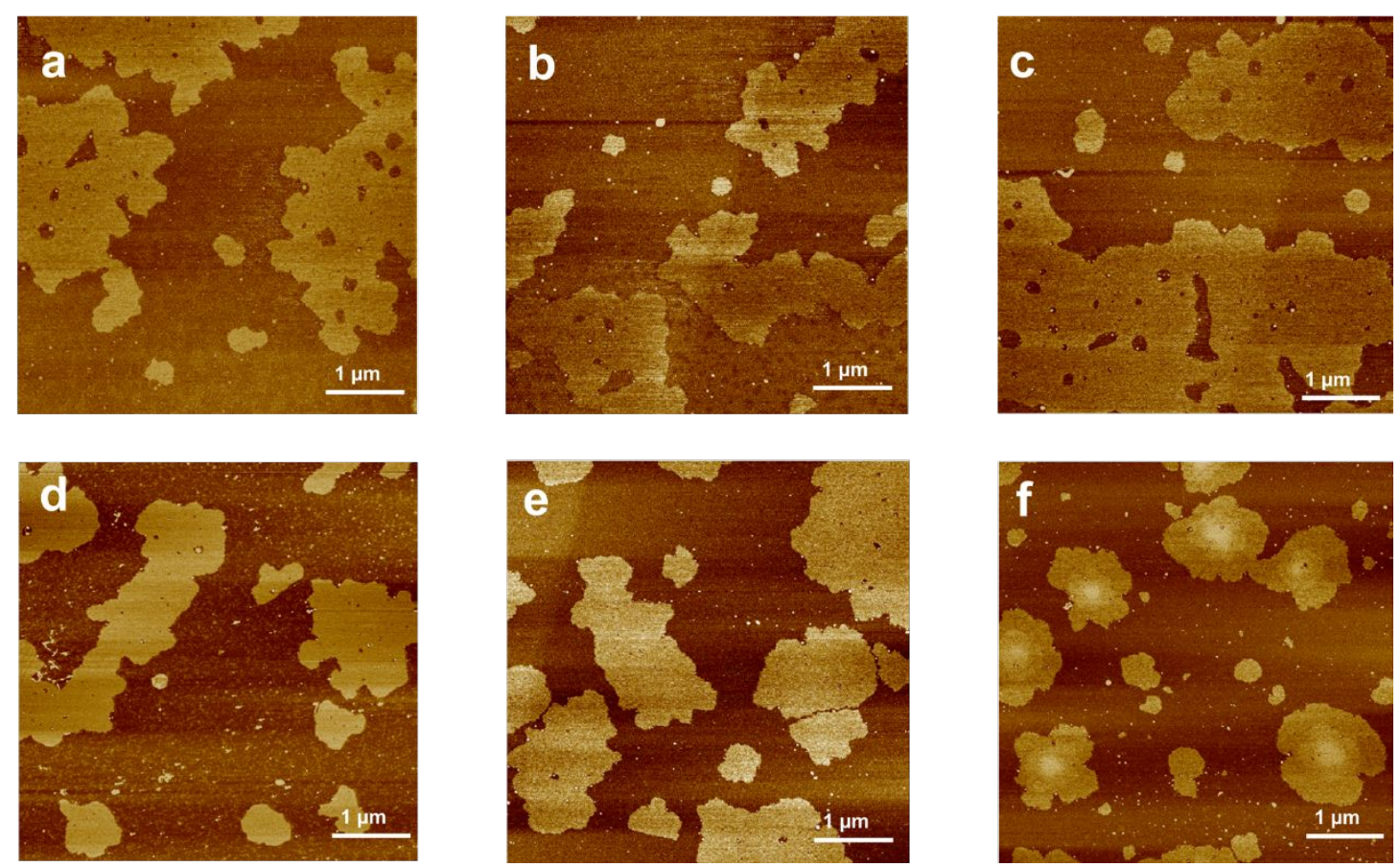

Figure S3. AFM images of COF-1.1 (a) (b) (c) and COF-1.3 (d) (e) (f) exfoliated in presence of CTAB. Shorter or longer exfoliation time usually result in different size and thickness of COF nanosheets. In the present study, ultrasonic exfoliation time less than 1 hour shows no extra defects are introduced in the COF nanosheets. The nonuniformity in thickness may cause the different COF-DNA interaction. Thicker COF nanosheet may have stronger interaction with DNA but decrease the spatial resolution as well. More precisely preparing COF nanosheets with a monolayer would be greatly helpful for further application of COF nanopores. 

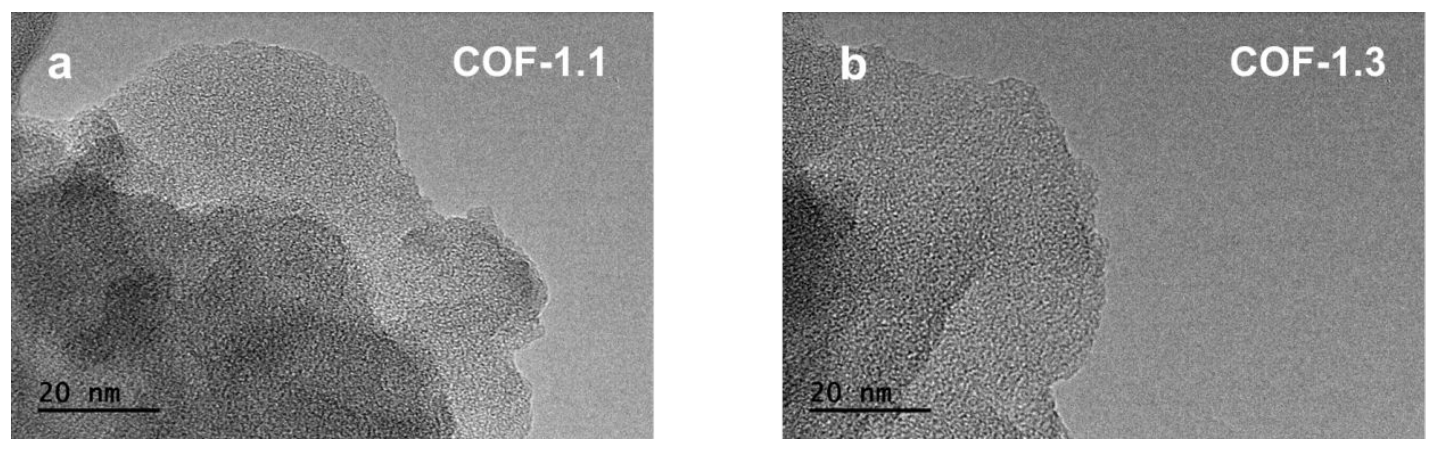

Figure S4. TEM images of COFs-1.1 (a) and COF-1.3 (b) exfoliated in presence of CTAB. 
COF-1.1
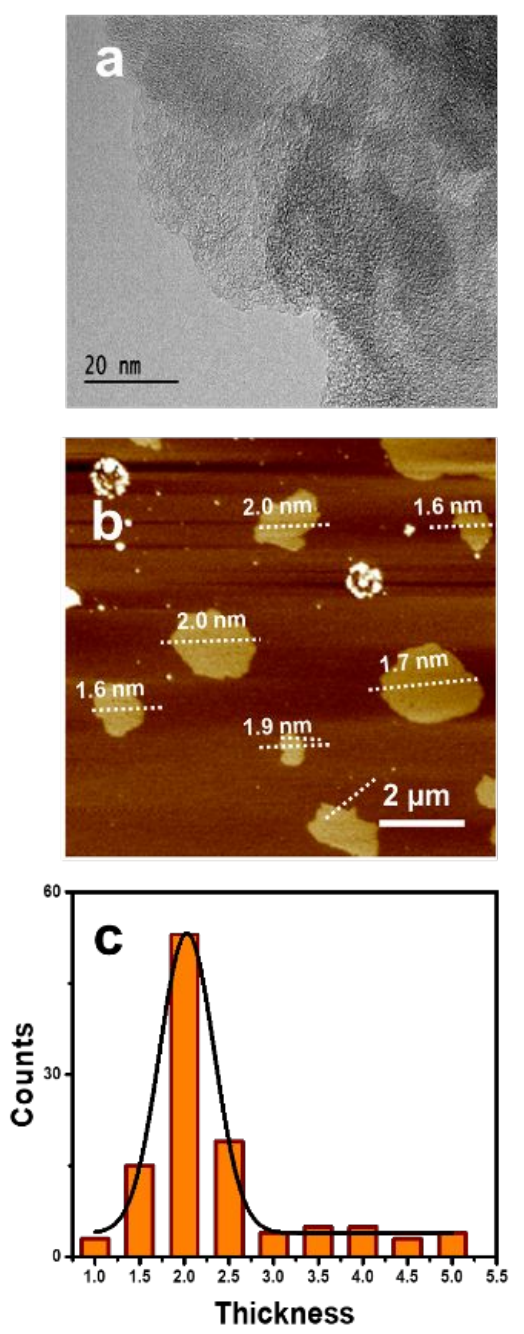

COF-1.3
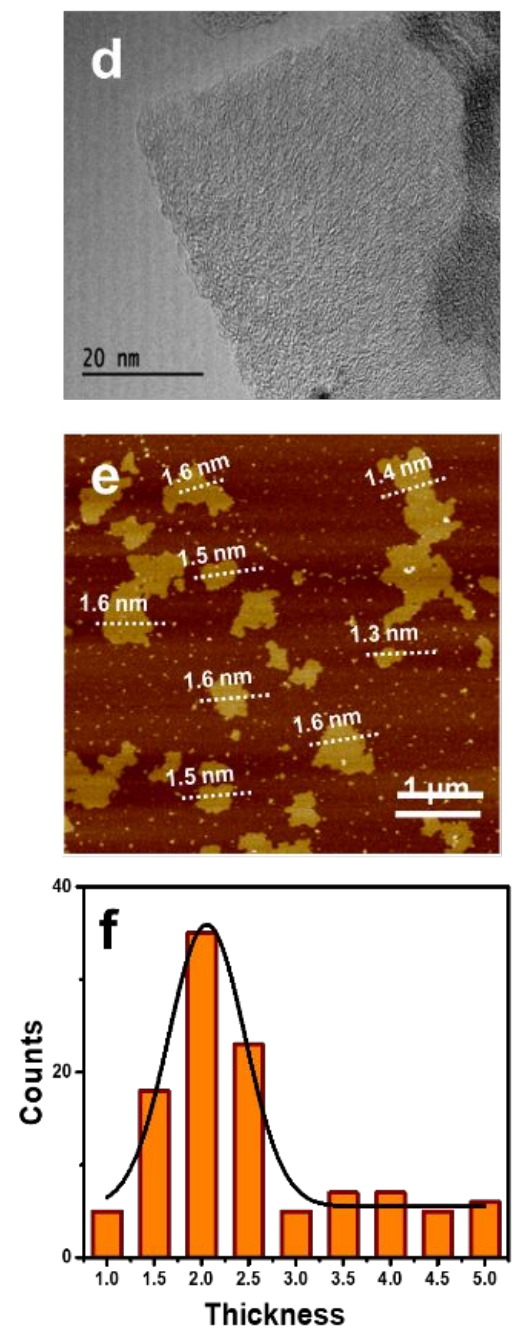

Figure S5. TEM (a, d) and AFM (b, e) images of 2D COF nanosheets exfoliated in the absence of CTAB. (c) and (f) are thickness statistics of COF-1.1 and CONF-1.3, respectively. The thicknesses of COFs exfoliated in the absence of CTAB are 2.0 \pm 0.3 and $2.1 \pm 0.4 \mathrm{~nm}$ for COF-1.1 and COF-1.3, respectively. 

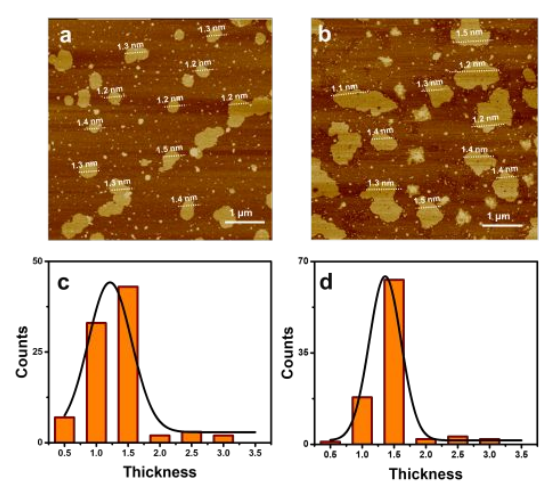

Figure S6. AFM images of COF-1.1 (a) and COF-1.3 (b) exfoliated in presence of CTAB. Both samples were dipped in pure water for $3 \mathrm{~min}$ to remove CTAB. Thickness statistics of COF-1.1 (c) and COF-1.3 (d). The thicknesses of COF nanosheets exfoliated in presence of CTAB are $1.2 \pm 0.3 \mathrm{~nm}$ and $1.14 \pm 0.2 \mathrm{~nm}$ for COF-1.1 and COF-1.3, respectively.

The average thicknesses of COF nanosheets exfoliated in the absence of CTAB are $2.0 \pm 0.3$ and $2.1 \pm 0.4 \mathrm{~nm}$ for COF-1.1 and COF-1.3, respectively (Fig. S5). The zeta potential of COF nanosheets obtained without adding CTAB fluctuated violently in a few hours after exfoliation accompanying the restacking process. As a comparison, COF nanosheets obtained in the presence of CTAB show a stable zeta potential of $+30 \pm 2 \mathrm{mV}$ after centrifugal washing with $10 \mathrm{mM} \mathrm{KCl}$ twice. However, centrifugal washing more than twice will cause restacking of COF nanosheets. Then, the prepared COF nanosheets can be stored at room temperature for more than 1 week and only need modest sonication before subsequent assembly. Similar surfactant-assisted method has been used in the anisotropic growth of MOF and formation of ultrathin MOF nanosheets. Here, the hydrophobic ends of a few CTAB molecules may dynamically attached on the COF skeletons while the hydrophilic ends stretching to the aqueous phase. Interestingly, CTAB did not obviously increase the thickness of the COF nanosheets. After removing CTAB by dipping the AFM samples in pure water for $3 \mathrm{~min}$, the thicknesses of COF nanosheets showed no significant changes (Fig. S6). This might suggest that few CTAB molecules attach to COF nanosheets. 

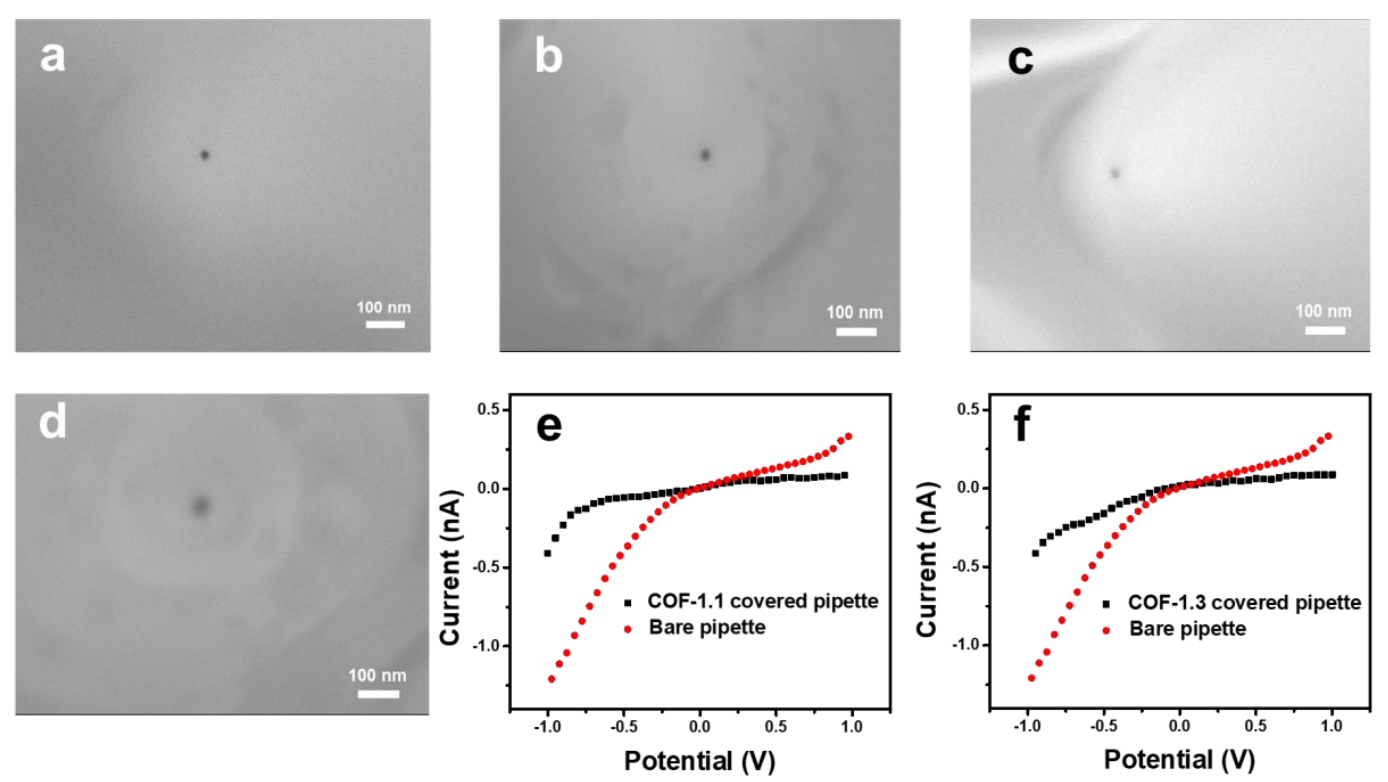

Figure S7. SEM images of bare quartz nanopipette (a-d). Current-voltage (I-V) curves obtained for a bare nanopipette and COF nanosheets-covered pipettes in $10 \mathrm{mM} \mathrm{KCl}$ solution. Scan rate, $0.05 \mathrm{~V} / \mathrm{s}$. COF-1.1 covered pipette (e). COF-1.3 covered pipette (f). 

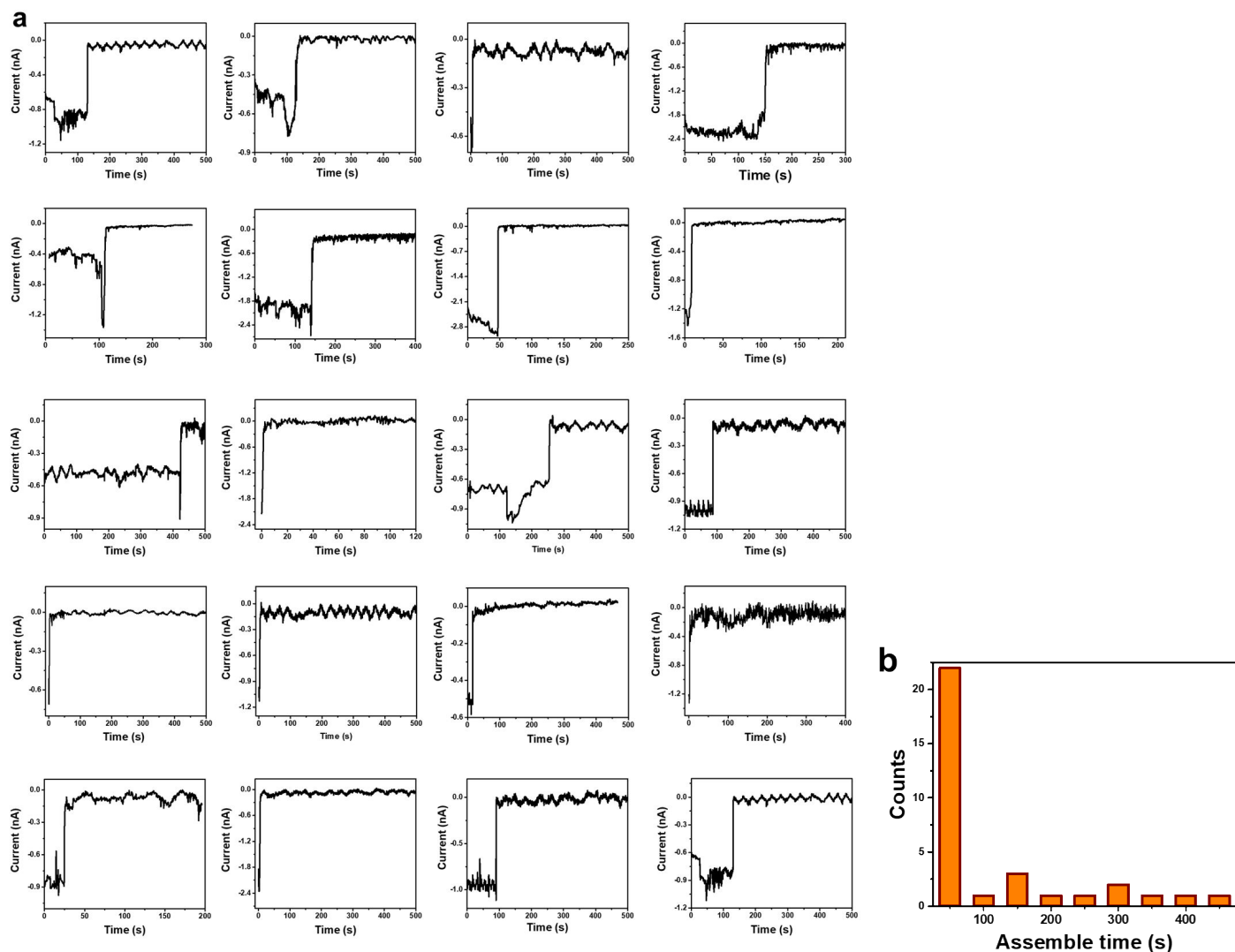

Figure S8. (a) 20 I-t curves of different nanopipettes recorded during the electrophoresis in $0.1 \mathrm{mg} / \mathrm{mL}$ COF-1.3 solution with a bias potential of $-1 \mathrm{~V}$. The current waving $(\sim 0.2$ nA) before and after the sudden current drop is caused by the environment vibration and a poor electromagnetic screen during the routine assembly. For DNA translocation experiments, a better Faraday cage and a vibration isolation table were used, and the current waving disappeared. (b) Histogram distribution of current drop time.

More than $90 \%$ of the current drops appear within 200 s, revealing that COF nanosheets assembled efficiently during the electrophoresis. Because such significant current drop only appears once for each bare nanopipette, we deduce that COF nanosheets cannot be stacked due to the repulsion force between them. However, the above current drop could hardly be observed when COF nanosheets were prepared in the absence of CTAB, revealing the great promotion of assembly process by positive charges from CTAB. 

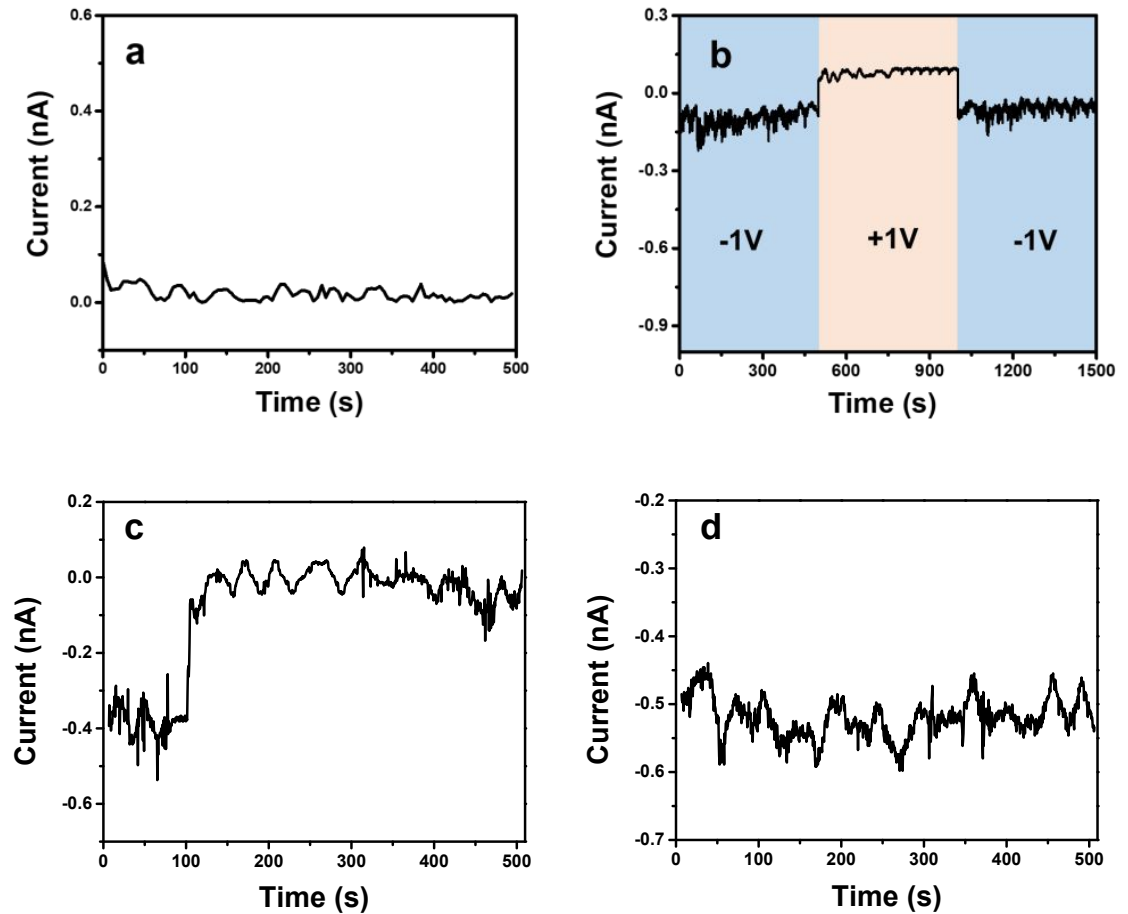

Figure S9. (a) I-t curve recorded directly after COF nanosheets assembled at the tip of the quartz nanopipette. A reverse bias potential of $+1 \mathrm{~V}$ was applied for $500 \mathrm{~s}$ and the current did not increase, showing the COF nanosheets won't become detached from the nanopipette. (b) I-t curve recorded in a solution without COF nanosheets after the COF assembly step. The quartz nanopipette was transferred into solution containing $10 \mathrm{mM} \mathrm{KCl}$ alone. The bias voltage was kept at each steps for $500 \mathrm{~s}(-1 \mathrm{~V}$ to $+1 \mathrm{~V}$ and back to $-1 \mathrm{~V})$ and the current was kept the same with the initial current level. Both curves showed the COF won't become detached from the nanopipette by applying $+1 \mathrm{~V}$ potential. (c) I-t curve of nanopipette recorded during the electrophoresis in $0.1 \mathrm{mg} / \mathrm{mL} \mathrm{COF}-1.3$ and $10 \mathrm{mM} \mathrm{KCl}$ with a bias potential of $-1 \mathrm{~V}$. The initial current is about $0.5 \mathrm{nA}$. (d) I-t curve of the same COF-nanopipette in $10 \mathrm{mM} \mathrm{KCl}$ after being treated with $1 \mathrm{mM} \mathrm{HCl}$ for 3 mins.

Such a firm attachment between COF nanosheets and nanopipette may initially originate from the amphiphilic CTAB, which binds the COF nanosheets with its hydrophobic end and the negatively charged quartz nanopipette with its amine group, 
respectively. Because the adsorption of $\mathrm{CTAB}$ on $\mathrm{COF}$ is reversible, when the $\mathrm{COF}$ covered nanopipette is dipped in DNA detection solution without CTAB, the trace amount of CTAB may diffuse into the bulk solution. Then the strong Van der Waals force dominates the COF-nanopipette interaction.

We further studied the Van der Waals force between COF and quartz nanopipette by dipping the COF-nanopipette device in $1 \mathrm{mM} \mathrm{HCl}$ solution $(\mathrm{pH}=3)$ for $3 \mathrm{~min}$ and measure the I-t curve again. As can be seen in Figure S9 $\mathrm{c}$ and d, the ionic current passing through the COF-nanopipette recovered to the initial current after the treatment, showing that the COF fall off from the nanopipette. This experiment confirmed that the Van der Waals force between $\mathrm{COF}$ and quartz is mainly induction force. Once the charge of quartz decrease under $\mathrm{pH} \mathrm{3}$, the induction force decreases and the COF cannot attach on the quartz nanopipette anymore. 
<smiles>CCN=c1cc2oc3cc(NCC)c(C)cc3c(-c3ccccc3C(=O)OCC)c-2cc1C</smiles><smiles>CN(C)c1ccc2c(-c3ccc(N4C(=O)C=CC4=O)cc3C(=O)[O-])c3ccc(=[N+](C)C)cc-3oc2c1</smiles>
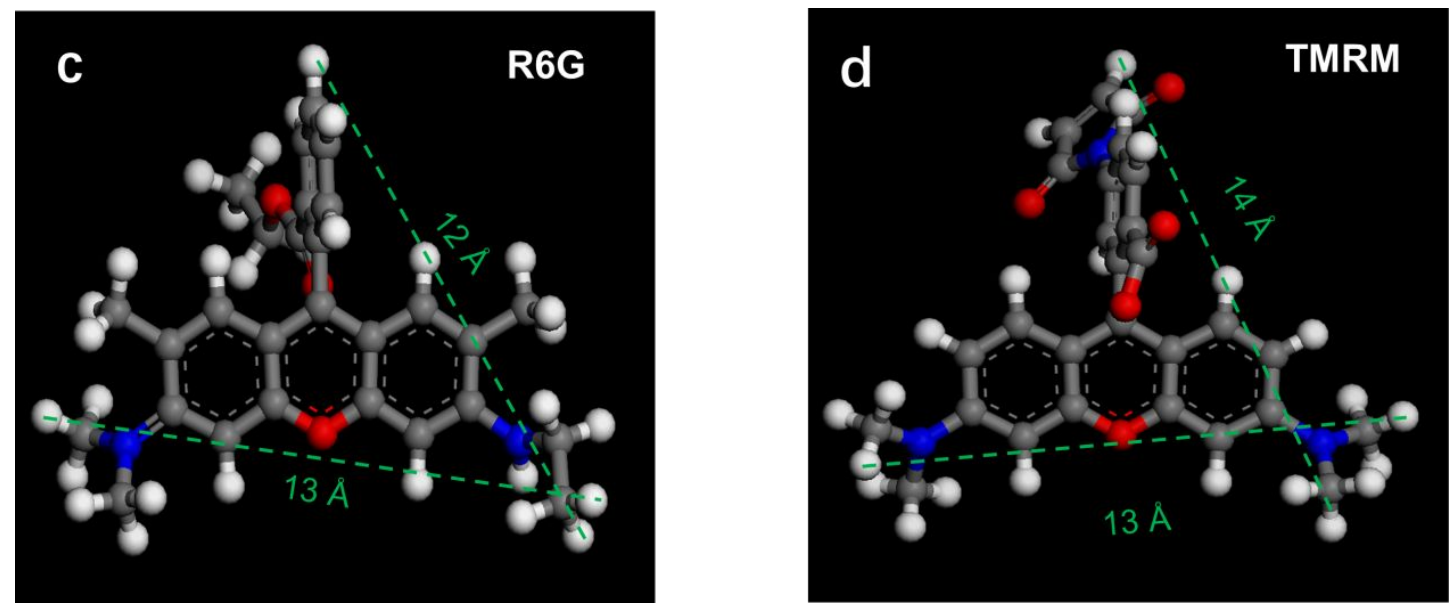

Figure S10. Chemical structure and size of the R6G (a, c) and TMRM (b, d). Both R6G and TMRM are in hydrochloride form and have the same backbone structure, and their side chains have similar polarity. Therefore, the different translocation behavior of R6G and TMRM through COF-1.3 are mostly caused by the pore size. 

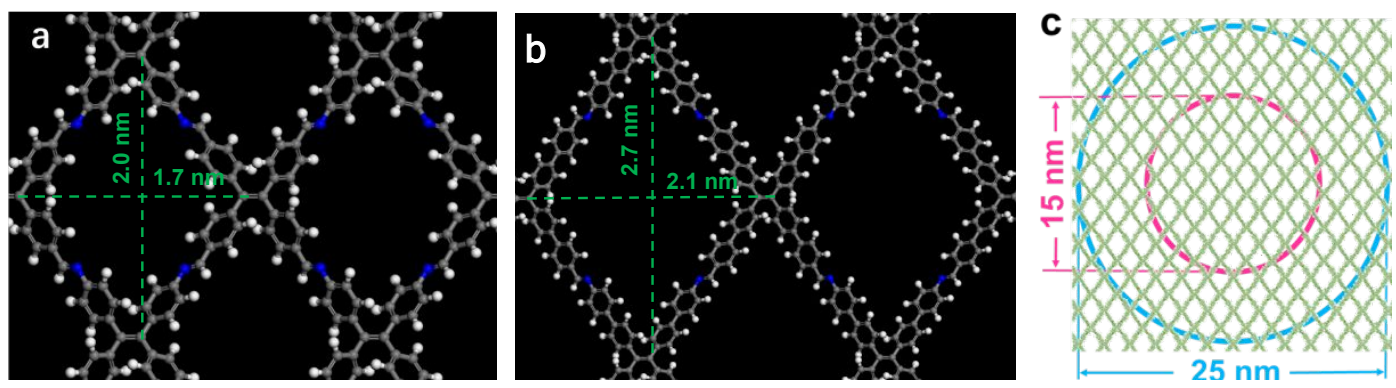

Figure S11. The size and effective area of the nanopores. (a) The effective area of a nanopore in COF-1.1 is $(1.7 \times 2.0) / 2=1.7 \mathrm{~nm}^{2}$. (b) The effective area of a nanopore in COF1.3 is $(2.7 \times 2.1) / 2=2.8 \mathrm{~nm}^{2}$. (c) Schematic illustration of COF-1.3 covering the nanopipette.

Considering the size of the COF monomers, each nanopore corresponds to an area about $1.7 \mathrm{~nm}^{2}$ in the COF-1.1 and $2.8 \mathrm{~nm}^{2}$ in the COF-1.3, respectively (Fig. S11a, b). Fig. S11c schematically illustrated how the number of nanopores within the orifice was estimated. Because of the electrostatic repulsion force between the negatively charged quartz and the DNA chains, the COF nanopores that allow DNA transport in subsequent analysis are more likely located at the central part of the orifice. We compared the relative standard deviation (RSD) of the baseline $(\sim 1.1 \%)$ and blockade current $(\sim 4.3 \%)$ and fund that the RSD of DNA translocation events is a little higher than baseline. This increased RSD may partially originate from the random passage of DNA through different nanopores. The only way to decrease the effect of the multi-nanopore is to fabricate COF-quartz nanopipette device with smaller orifice. 

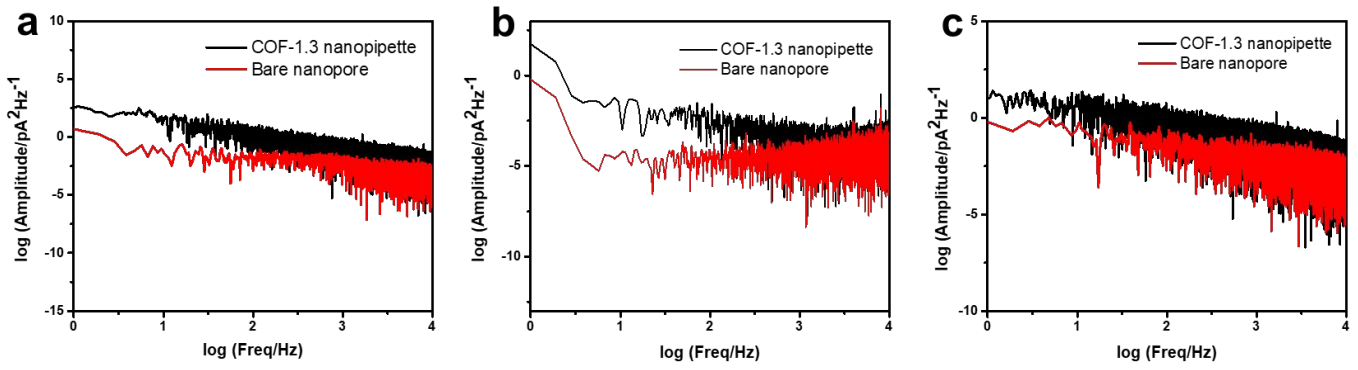

Figure S12. Comparison of the power spectra density of the COF-1.3 nanosheet-covered nanopipette at voltage bias of (a) $300 \mathrm{mV}$, (b) $400 \mathrm{mV}$ and (c) $500 \mathrm{mV}$, respectively. All the data were obtained at a sampling frequency of $500 \mathrm{kHz}$ and were filtered at $10 \mathrm{kHz}$. 

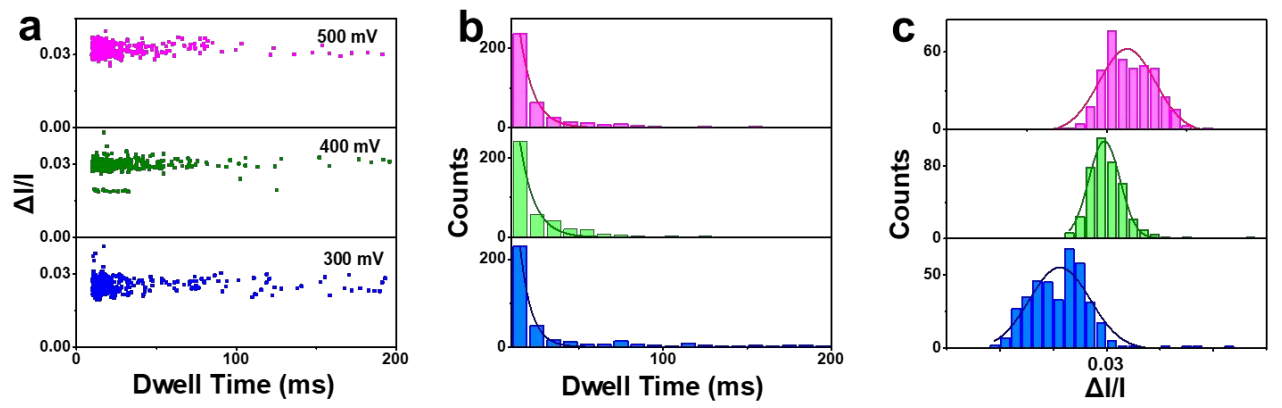

Figure S13. The transport of ct-ssDNA $(0.67 \mathrm{nM})$ through bare quartz nanopore in $1 \mathrm{M}$ $\mathrm{KCl}$ solution (10 mM Tris-HCl, 1 mM EDTA, pH 8.0). (a) Scatterplots of fractional current blockades vs. dwell time for ct-DNA going through COF-1.3 at the indicated applied voltages. (b) Dwell-time distributions for COF-1.3 along with the fits (solid lines) at various voltages in the same range as in (a). (c) Fractional current amplitude distributions of the bare quartz nanopitette fit Gaussian distribution. 


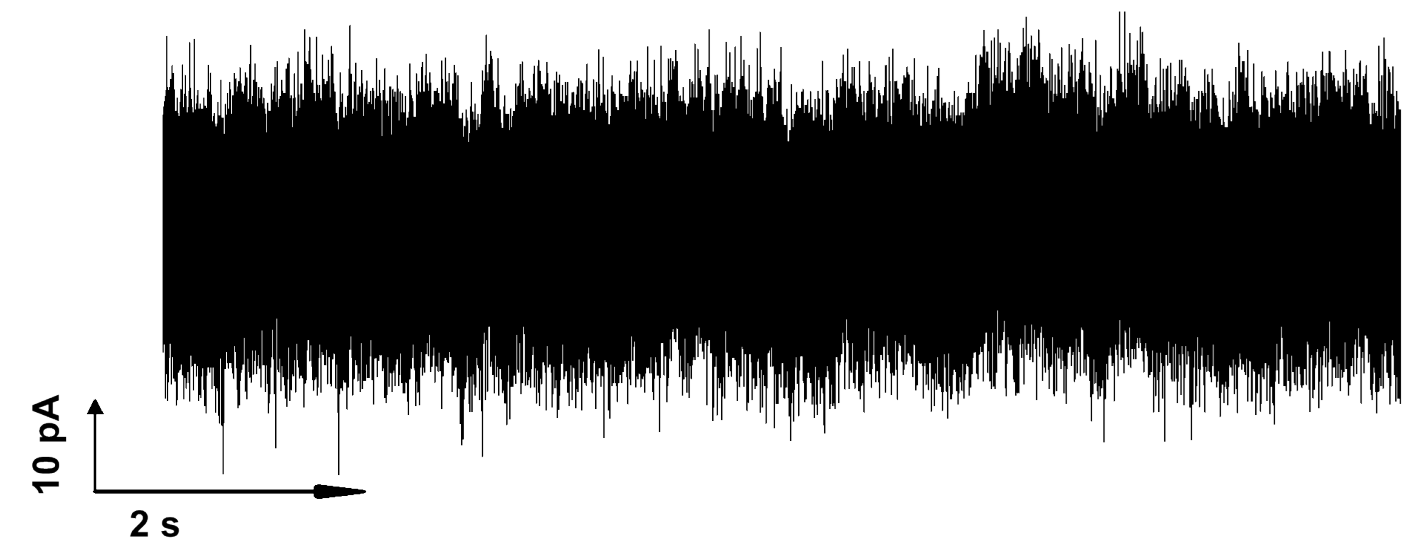

Figure S14. The transport of ct-DNA through COF-1.1 nanopores. No current drops were observed in this $10 \mathrm{~s}$ current trace. 


\section{S3. Supplementary Tables}

Table S1. The sequence of DNA-80.

\begin{tabular}{|l|l|}
\hline Construct & \multicolumn{1}{|c|}{ Sequence } \\
\hline DNA-80 & $\begin{array}{l}\text { TAGCTTATCAGACTGATGTTGAACTTTAGAACTTTAGAACTT } \\
\text { TTCAGATCTCACTATCGCATTCTCATGCAGGTCGTAGC }\end{array}$ \\
\hline
\end{tabular}


Table S2. Comparison of DNA translocation speed and capture rate of nanopore membranes based on 2D materials.

\begin{tabular}{|c|c|c|c|c|c|c|}
\hline $\begin{array}{c}\text { Type of 2D } \\
\text { material }\end{array}$ & Diameter & DNA type & $\begin{array}{c}\text { Potential } \\
(\mathrm{mV})\end{array}$ & $\begin{array}{c}\text { Translocation } \\
\text { speed ( } \mu \text { s/base) }\end{array}$ & $\begin{array}{c}\text { capture rate } \\
\left(\mathrm{s}^{-1} \mathrm{nM}^{-1}\right)\end{array}$ & Ref. \\
\hline \multirow[t]{3}{*}{ graphene } & 22 & dsDNA & 200 & 0.056 & not reported & S5 \\
\hline & 8 & $\begin{array}{l}15 \mathrm{kbp} \\
\text { dsDNA }\end{array}$ & 100 & $\sim 0.005$ and 0.03 & 1 & S6 \\
\hline & 5 & $\begin{array}{l}\text { 20-mer } \\
\text { ssDNA }\end{array}$ & 500 & 8.5 & not reported & S7 \\
\hline $\mathrm{WS}_{2}$ & $4.4 \pm 0.9$ & $\begin{array}{l}\text { 15kbp- } \\
\text { dsDNA }\end{array}$ & 400 & 0.041 & not reported & S8 \\
\hline \multirow[t]{2}{*}{ h-BN } & $\sim 6$ & $\begin{array}{l}\text { 10kbp- } \\
\text { dsDNA }\end{array}$ & 160 & 0.016 & not reported & S9 \\
\hline & 4 & $\begin{array}{l}\text { 48.5kbp- } \\
\text { dsDNA }\end{array}$ & 150 & 0.062 & not reported & S10 \\
\hline \multirow[t]{3}{*}{$\mathrm{MoS}_{2}$} & 20 & dsDNA & 200 & $\sim 0.022$ & not reported & S11 \\
\hline & 2.3 & ssDNA & 200 & 0.1 & 0.95 & S12 \\
\hline & 20 & dsDNA & 400 & $20-1000$ & not reported & S13 \\
\hline MXene & 7.2 & dsDNA & 500 & 2 & 0.042 & S14 \\
\hline $\mathrm{COF}$ & 1.1 & ssDNA & $200-400$ & 270 & 3.41 & $\begin{array}{l}\text { This } \\
\text { study }\end{array}$ \\
\hline
\end{tabular}




\section{Reference}

S1. Bunck, D. N.; Dichtel, W. R. Bulk Synthesis of Exfoliated Two-Dimensional Polymers

Using Hydrazone-Linked Covalent Organic Frameworks. J. Am. Chem. Soc. 2013, 135, 14952-14955.

S2. Wang, S.; Wang, Q. Y.; Shao, P. P.; Han, Y. Z.; Gao, X.; Ma, L.; Yuan, S.; Ma, X. J.; Zhou, J. W.; Feng, X.; Wang, B. Exfoliation of Covalent Organic Frameworks into FewLayer Redox-Active Nanosheets as Cathode Materials for Lithium-Ion Batteries. J. Am. Chem. Soc. 2017, 139, 4258-4261.

S3. Clark RJH and Hester RE (eds), Biomolecular Spectroscopy, Vol. 20, Part A, Wiley, New York, 1993.

S4. Colthurp NB, Daly LH and Wiberley SE, Introduction to infrared and Raman spectroscopy, Academic Press, Boston, 1990.

S5. Schneider, G. F.; Kowalczyk, S. W.; Calado, V. E.; Pandraud, G.; Zandbergen, H. W.; Vandersypen, L. M.; Dekker, C., DNA translocation through graphene nanopores. Nano Lett. 2010, 10, 3163-3167.

S6. Merchant, C. A.; Healy, K.; Wanunu, M.; Ray, V.; Peterman, N.; Bartel, J.; Fischbein, M. D.; Venta, K.; Luo, Z.; Johnson, A. T.; Drndic, M., DNA translocation through graphene nanopores. Nano Lett. 2010, 10, 2915-2921.

S7. Deng, Y.; Huang, Q.; Zhao, Y.; Zhou, D.; Ying, C.; Wang, D., Precise fabrication of a $5 \mathrm{~nm}$ graphene nanopore with a helium ion microscope for biomolecule detection. Nanotechnology 2016, 28, 045302.

S8. Danda, G.; Masih Das, P.; Chou, Y.-C.; Mlack, J. T.; Parkin, W. M.; Naylor, C. H.; Fujisawa, K.; Zhang, T.; Fulton, L. B.; Terrones, M.; Johnson, A. T. C.; Drndić, M., Monolayer WS2 Nanopores for DNA Translocation with Light-Adjustable Sizes. ACS Nano 2017, 11, 1937-1945.

S9. Liu, S.; Lu, B.; Zhao, Q.; Li, J.; Gao, T.; Chen, Y.; Zhang, Y.; Liu, Z.; Fan, Z.; Yang, F.; You, L.; Yu, D., Boron Nitride Nanopores: Highly Sensitive DNA Single-Molecule 
Detectors. Adv. Mater. 2013, 25, 4549-4554.

S10. Zhou, Z.; Hu, Y.; Wang, H.; Xu, Z.; Wang, W.; Bai, X.; Shan, X.; Lu, X., DNA Translocation through Hydrophilic Nanopore in Hexagonal Boron Nitride. Sci. Rep 2013, 3,3287 .

S11. Liu, K.; Feng, J.; Kis, A.; Radenovic, A., Atomically Thin Molybdenum Disulfide Nanopores with High Sensitivity for DNA Translocation. ACS Nano 2014, 8, 2504-2511. S12. Ivankin, A.; Henley, R. Y.; Larkin, J.; Carson, S.; Toscano, M. L.; Wanunu, M., Label-Free Optical Detection of Biomolecular Translocation through Nanopore Arrays. ACS Nano 2014, 8, 10774-10781.

S13. Feng, J.; Liu, K.; Bulushev, R. D.; Khlybov, S.; Dumcenco, D.; Kis, A.; Radenovic, A., Identification of single nucleotides in MoS2 nanopores. Nat. Nanotechnol. 2015, 10, 1070-1076.

S14. Mojtabavi, M.; VahidMohammadi, A.; Liang, W.; Beidaghi, M.; Wanunu, M., SingleMolecule Sensing Using Nanopores in Two-Dimensional Transition Metal Carbide (MXene) Membranes. ACS Nano 2019, 13, 3042-3053. 